\title{
NOTIZEN
}

\section{Die Ionosphäre während der partiellen Sonnen- finsternis am 28. April 1949}

\author{
Von Dietrich Stranz
}

(Z. Naturforschg. 5 a, 172-173 [1950]; eingeg. am 15. Sept. 1949)

Am Morgen des 28. April 1949 fand eine partielle Sonnenfinsternis statt, die nach den Angaben des Nautical Almanac 1949 um 05 $51.8 \mathrm{~m}$ GMT in $\lambda=+5^{\circ} 20^{\prime}$ und $\varphi=+25^{\circ} 15^{\prime}$ begann, um $07 \mathrm{~h} 48.4 \mathrm{~m}$ in $\lambda=+55^{\circ} 56^{\prime}$ und $\varphi=+62^{\circ} 04^{\prime}$ ihre Maximalphase hatte und um $09^{\mathrm{h}} 44.5^{\mathrm{m}}$ in $\lambda=-165^{\circ} 55^{\prime}$ und $\varphi=+69^{\circ} 40^{\prime}$ endete. Die Konjunktion fand um $08 \mathrm{~h} 52 \mathrm{~m} 50.5^{\mathrm{s}}$ statt. Rektaszension und Deklination der Sonne waren zu diesem Zeitpunkt ${ }^{\prime} \odot=2^{\mathrm{h}} 21^{\mathrm{m}} 29.5^{\mathrm{s}}$ und $\delta \odot=+14^{\circ} 05^{\prime} 42.4^{\prime \prime}$, während der Mond mit $\delta=+15^{\circ} 17^{\prime} 57.4^{\prime \prime}$ nördlich der Sonne vorbeizog.

Für Götebarg dauerte die Phase der Verfinsterung von $06^{\mathrm{h}} 36^{\mathrm{m}}$ bis $08^{\mathrm{h}} 31^{\mathrm{m}} \mathrm{GMT}$ mit dem Maximum (0.37) um $07 \mathrm{~h} 32 \mathrm{~m}$. Sieht man sich für diesen Morgen die Grenzfrequenzen der E- und F-Schicht an, so findet man eine leichte Verzögerung im Anstieg der Ladungsträgerdichte in der E-Schicht während der Verfinsterungsphase, die nach Verlassen des Mondschattens sehr schnell wieder eingeholt wird. Gegen 09h GMT ist der normale Wert von $1,6 \cdot 10^{5} \mathrm{~cm}^{-3}$ entspr. $3,6 \mathrm{MHz}$ wieder erreicht. Daran ist nichts Bemerkenswertes, und in früheren Arbeiten ist darüber schon berichtet worden 1 .

Auffällig und unerwartet in ihrem Verhalten ist hingegen die F-Schicht (Abb. 1), deren Trägerdichte von Sonnenaufgang an, also schon 2 Stdn. vor Einsetzen der Verfinsterung am Beobachtungsort, ungewöhnlich rasch ansteigt, so daß zur Maximalphase die Grenzfrequenz um ganze $4 \mathrm{MHz}$ über dem Zentralwert (median) des Monats liegt. Gegenüber den folgenden Tagen ist die Abweichung sogar noch größer. Lindau (Harz) zeigt einen ganz ähnlichen Effekt, die höchste positive Abweichung beträgt dort ebenfalls zur maximalen Verfinsterungsphase $3 \mathrm{MHz}$. Leider sind in Tromsö die Messungen erst ab 08h GMT vorhanden. Die Grenzfrequenzen lagen hier aber auch relativ hoch; der Effekt war jedoch nicht so ausgeprägt.

Die positive Abweichung hält sich in Göteborg, auffälliger als in Lindau, bis nach $10^{\mathrm{h}} \mathrm{GMT}$, um gegen $12^{\mathrm{h}}$ bzw. $10.30^{\mathrm{h}}$ wieder auf Null gekommen zu sein. Es ist natürlich sehr verlockend, dieses merkwürdige Zusammenfallen der Zunahme der Trägerdichte der F-Schicht (von $1 \cdot 10^{6}$ auf rund das Doppelte!) mit der partiellen Sonnenfinsternis irgendwie in Zusammenhang zu bringen, wenn auch eine Erklärung nicht ohne weiteres auf der Hand liegt.

Ein direkter Einfluß des Mondschattens (Abkühlungseffekt) ist unmöglich schon 2 Stdn. vor und noch 2 Stdn. nach der Finsternis verständlich. Man könnte höchstens die Abschirmung von Teilen der obersten Korona (im Abstand von etwa $1^{\circ}$ von der Sonne) annehmen, wodurch

- Box 123, Askim, Schweden.

1 Monthly Notices Roy. Astronom. Soc. 98, 666 [1938]. das photochemische Gleichgewicht der F-Schicht in dem beobachtcien Sinne aus unbekanntem Anlaß gestört werden konnte. Es sei vermerkt, daß Kiepenheuer eine negative Korrelation zwischen der Grenzfrequenz der F-Schicht und der Koronahelligkeit fand.

Beim Eintritt der Verfinsterung in Göteborg wurde ein kurzer Mögel-Dellinger-Effekt beobachtet, bei dem minutenlange Echos von etwa $87 \mathrm{~km}$ Höhe

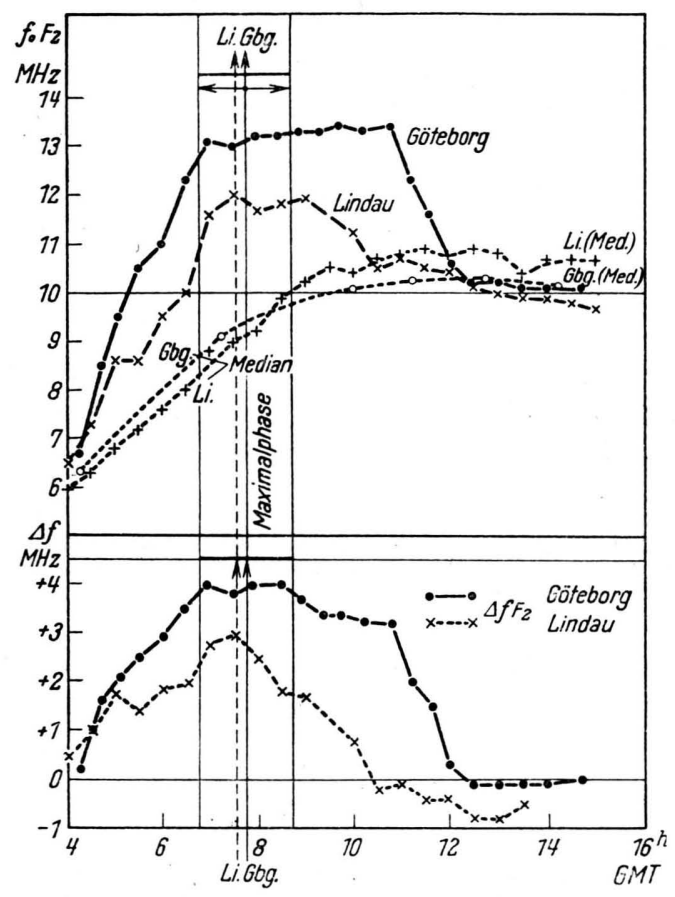

Abb. 1. Grenzfrequenz und Abweichung vom Zentralwert in der F-Schicht. 28. April 1949 und April 1949.

(D-Schicht) auf 3 und $5,8 \mathrm{MHz}$ auftraten. Ob eine Begünstigung dieser tiefliegenden Echos durch die besonderen Umstände in der Ionosphäre vorlag, kann nicht ohne weiteres entschieden werden. Aber es ist denkbar, daß die teilweise Abdeckung der Korona den Temperaturanstieg in der F-Schicht durch Verminderung eines entscheidenden Photoprozesses in einem solchen Maße verhinderte, daß die normale Dichteabnahme der Luft nach oben gestört war und somit Anlaß zu dem raschen Ladungsträgeranstieg im Niveau der D-Schicht (großer Gradient) während der chromosphärischen Eruption gab. Im selben Sinne könnte die vorübergehende "winterliche“ Temperaturverteilung in der F-Schicht deren unverhältnismäßig hohe Grenzfrequenz kurz vor, während und kurz nach der Verfinsterungsphase erklären.

Man muß über dem Schattengebiet geringeren Luftdruck in der Höhe der F-Schicht annehmen, so daß ein Transport von Ladungsträgern von außen heran aus südlicheren Breiten stattfinden wird. 
Die Messungen in Göteborg wurden am Institut für Elektronik, Chalmers Technische Hochschule, durchgeführt. Die Werte von Lindau (Harz) und Tromsö wurden in dankenswerter Weise von den dortigen Instituten zur Verfügung gestellt.

\section{Die Planckschen Maßeinheiten}

Von Hans Joachim Rodewald

Physikalisches Institut der Techn. Universität Berlin

(Z. Naturforschg. 5 a, 173 [1950]; eingeg. am 1. Febr. 1950)

In der physikalischen Kosmologie genügen die Systeme Welt, Galaxie, Stern und Elementarteilchen mit guter Annäherung den hierarchischen Beziehungen

$$
M_{i} / M_{i+1}=\imath^{1 / 4} ; \quad R_{i} / R_{i+1}=i^{1 / 8} ; \gamma=10^{40},
$$

sofern man diesen Systemen die Indices 0, 1, 2 und 8 zuordnet. Die einzige grobe Abweichung (beim Sternradius) verschwindet, wenn man als Radius den Wirkungsradius einsetzt, d. h. die Grenzentfernung, in der ein System noch befähigt ist, auf Grund seiner Gravitation seine Massen zusammenzuhalten ${ }^{1}$. Wählt man für $M_{8}$ die Neutronenmasse, wie es die Charliersche Instabilitätsbedingung 2 verlangt, und für $R_{8}$ die Compton-Wellenlänge $\boldsymbol{h} / \boldsymbol{M}_{8} c_{0}$ des Neutrons, was durch experimentelle Ergebnisse und die Mesonentheorie der Kernkräfte nahegelegt wird, so ergibt sich die Konstante $A$ in der Gleichung

$$
f M_{0} / R_{0}=A c_{0}{ }^{2}
$$

mit $1 \%$ Genauigkeit zu $3 \pi$. Die Gleichung wie auch die Konstante $3 \pi$ ist dabei kein Fremdbestandteil. C h a r li e r leitete sie in korrespondierender Form mit Hilfe des Virialsatzes für die Periode der Galaxie ab (a. a. O. Gleichung 20):

$$
T_{1}=\sqrt{3 \pi / f \mu_{1}},
$$

$$
T_{1}=R_{1} / c_{1}, \mu_{1}=M_{1} / R_{1}^{3}=\text { Dichte der Galaxie. }
$$

Bringt man die hierarchischen Beziehungen in die Form

$$
R_{i} / R_{i+x}=\sqrt{ } M_{i} / M_{i+x}=\gamma^{\mathrm{x} / 8},
$$

so erhält man die für den hierarchischen Materieaufbau charakteristische Grundgleichung (2), wobei allerdings die Instabilitätsbedingung in die kritischen Werte der Komponenten verlegt worden ist. In Verbindung mit (1) liefert die Grundgleichung (2) das folgende System natürlicher Maßeinheiten:

$$
\begin{aligned}
& M_{8}=\sqrt{3 \pi c_{0} h / f \gamma=1,675 \cdot 10^{-24} \mathrm{~g},} \\
& R_{8}=\sqrt{f h \gamma / 3 \pi c_{0}{ }^{3}}=1,319 \cdot 10^{-13} \mathrm{~cm}, \\
& \tau_{8}=\sqrt{f h \gamma / 3+c_{0}{ }^{5}=4,400 \cdot 10^{-24} \mathrm{sec},} \\
& t^{s}=\frac{1}{k} \sqrt{3 \cdot \tau c_{0}{ }^{5} h / f_{\gamma}}=1,091 \cdot 10^{13} \mathrm{grad} .
\end{aligned}
$$

Diese unterscheiden sich von den P l a n c k schen Maßeinheiten durch den Faktor $/ 3 \pi / \gamma$ bzw. dessen Reziprokwert. In einer einfachen dimensionsanalytischen Betrachtung ergeben sie sich als Grundeinheiten, wenn man der Gravitationskonstanten den Wert $3 \pi / \gamma$ (anstatt 1 wie bei $\mathrm{Planck}$ ) erteilt und $c_{0}, h$ und $k$ zur Einheit macht. Da sie im Gegensatz zu den Planckschen Maßeinheiten eine reale Bedeutung besitzen $\left(\tau_{8}\right.$ ist die Schwingungsdauer der Compton-Welle, $t_{8}$ die Temperatur bei adiabatischer Verdampfung des Neutrons ${ }^{4}$ ), soll an einem Beispiel ihre Brauchbarkeit geprüft werden.

Die von Chandrasekhar thermodynamisch abgeleitete Formel für die Grenzmasse eines entarteten Sterns wird hier in Anlehnung an die von F. Hund und P. Jordan benutzte Fassung wiedergegeben:

$$
M_{\mathrm{St}}=\mathrm{const} \cdot M_{8}\left(c_{0} h / f M_{8}^{2}\right)^{3 / 2} .
$$

Nach Auflösung des Klammerausdrucks mittels (3) erhält man

$$
M_{\mathrm{St}}=\text { const } \cdot M_{8} \gamma^{3 / 2} .
$$

So gelangt man zu einer hierarchischen Beziehung, die in ähnlicher Form in der Eddingtonschen Theorie der hellsten Sterne, der Kotharischen Theorie der weißen Zwerge, der Zwickyschen Theorie des Neutronensterns und der Jordanschen Theorie der Sternentstehung wiederkehrt.

Wenn die vorliegenden Ausführungen auch nur zur Lösung eines speziellen physikalischen Problems beitragen sollten, so muß doch schon vorausgeschickt werden, daß die Hubble-Konstante im hierarchischen Weltbild keine Grundkonstante ist. Die Rotverschiebung der extragalaktischen Spiralen wird in Abweichung von den hierzu geäußerten Ansichten C. V. L. Charliers als Einsteinsche Rotverschiebung gedeutet, wobei die Entfernungslinearität erhalten bleibt und der Wert der Hubble-Konstanten sich im Modell zu

$$
\because=/ 3 \pi c_{0}^{5} / f h \gamma^{3}=2,273 \cdot 10^{-17} \mathrm{sec}^{-1}
$$

errechnet.

Eine ausführliche Darstellung der Theorie erfolgt in Kürze.

1 H. J. Rodewald, Z. Naturforschg. 4 a, 224 [1949].

2 C. V. L. C h a r li e r, Ark. Mat., Astronom. Fysik, Ser. I, Bd. 16, Nr. 22 (Medd. Lunds Astronom. Observ. Nr. 98) [1922].

3 M. P l a n c k, „Die Einheit des physikalischen Weltbildes“, Vortrag an der Universität Leiden am 9. 12. 1908; Vorlesungen über die Theorie der Wärmestrahlung, 2. Aufl., Leipzig 1913, § 164.

4 Daß Temperaturen von diesen Größenordnungen im Kosmos auftreten, ist durch grundlegende Untersuchungen von F. $\mathrm{H} \mathrm{u} \mathrm{n} \mathrm{d,} \mathrm{Ergebn.} \mathrm{exakt.} \mathrm{Naturwiss.} \mathrm{15,} 189$ [1936], P. J or d a n, Z. Naturforschg. 1, 301 [1946], und F. G. Houtermans u. J. H. D. J e n s e n, Z. Naturforschg. 2 a, 146 [1947], glaubhaft gemacht worden. 